\title{
Waveguide-mode Illumination for Enhanced Fluorescent Microscopy
}

\author{
Masato Yasuura and Makoto Fujimaki* \\ Electronics and Photonics Research Institute, National Institute of Advanced Industrial Science and Technology (AIST), \\ Central 5, 1-1-1 Higashi, Tsukuba, Ibaraki 305-8565, Japan
}

(Received July 2, 2018; accepted August 27, 2018)

Keywords: near-field light, fluorescent assay, immunoassay, total internal reflection

In this research, we apply the near field excited by waveguide mode (WGM) to the illumination of a total internal reflection fluorescence (TIRF) microscope. By utilizing the electric field enhancement by WGM, we can replace the laser light source with a lowoutput light source such as an LED. By measuring the reflection spectra of the WGM chip whose surface was covered with cells, the effect of the cell on the reflection spectra was evaluated. On the basis of the evaluation, we designed an asymmetric trapezoidal prism to excite two fluorescent dyes with different excitation wavelengths on a single WGM chip. By using the trapezoidal prism with different base angles to enhance each wavelength, enhanced fluorescence observations using two fluorescent dyes with a simple optical system became possible. In this optical system, the observation of cultured cells, whose cytoskeleton and nuclei were separately stained with two fluorescent dyes, was carried out. It was confirmed that the fluorescence of each dye was effectively enhanced at both wavelengths.

\section{Introduction}

Near-field illumination is utilized in surface observation, such as total internal reflection fluorescence (TIRF) microscopy. ${ }^{(1,2)}$ The TIRF microscope is suitable for measuring the topography of cell-substrate contacts. However, it requires a laser as a light source, and the apparatus tends to be expensive and large. There is a method of enhancing near-field light in order to apply TIRF even with a low-output light source such as an LED. A common method that uses surface plasmon resonance (SPR) for the amplification of light is called surfaceplasmon field-enhanced fluorescence spectroscopy (SPFS) or surface-plasmon field-enhanced fluorescence microscopy (SPFM), depending on the observation method. ${ }^{(3-5)}$ However, since a chip with a metal surface is used to excite SPR, methods of preventing the escape of the energy of fluorescence to the metal surface are required to suppress quenching. The use of the waveguide mode (WGM) is one of the methods of enhancing near-field light utilizing multiple reflections. ${ }^{(6,7)}$ When the wavelength of the incident light and the incident angle satisfy certain conditions, near-field light is enhanced on the total reflection surface. Unlike SPR, WGM can

*Corresponding author: e-mail: m-fujimaki@aist.go.jp

https://doi.org/10.18494/SAM.2019.2044 
use a chip surface with $\mathrm{SiO}_{2}$ glass, which is an insulator; thus, there is no quenching problem. In this research, we focus on the TIRF microscope that applies this WGM illumination.

Fluorescent dyes are often used to distinguish signals in fluorescence assays. ${ }^{(8)}$ When using several fluorescent dyes with different excitation wavelengths on a WGM chip, it is necessary to introduce excitation lights with different incident angles for each excitation wavelength. Simply replacing the light source is insufficient, and it is necessary to adjust the optical arrangement. The adjustment of an optical system inhibits seamless observations for multi-fluorescence imaging. Additionally, it is difficult to simplify a device. In our previous study, we have reported the optical arrangement of a parallel-incident-type WGM sensor, ${ }^{(7)}$ and we adopted the optical arrangement in this study. The optical arrangement is fixed so that light from the light source enters the prism at an angle parallel to the sensor chip, and the base angle of the prism is changed according to the wavelength to be enhanced. With the optical arrangement of this parallel-incident-type WGM sensor, it is possible to enhance the two-wavelength light on one WGM chip using an asymmetric trapezoidal prism, so that it can be easily formed into a simple device.

In this study, two-color fluorescence observations were carried out using two fluorescent dyes, an asymmetric trapezoidal prism, and two light sources of different wavelengths applied to the optical arrangement of a parallel-incident-type WGM sensor. Cultured cells were fixed on the WGM chip, and the nucleus and cytoskeleton were separately stained with fluorescent dyes and observed.

\section{Materials and Methods}

\subsection{Reagents}

Madin-Darby canine kidney cells (MDCK, European Collection of Authenticated Cell Cultures, Public Health England, UK) were used as a target. Dulbecco's Modified Eagle Medium (DMEM, 11885084, Thermo Fisher Scientific Inc., MA, USA) was used as a cell culture medium. Fetal bovine serum (FBS, Thermo Fisher Scientific Inc.) was used as an additive agent in the medium. Trypsin-ethylenediaminetetraacetic acid solution $[0.25 \%$ trypsin-EDTA(1X), 25200-056, Thermo Fisher Scientific Inc.] was used for cell dissociation. Phosphate-buffered saline (PBS) was prepared by diluting 10× PBS(-) (163-25265, Wako Pure Chemical Industries, Ltd., Japan) 10 times. Triton X-100 (Molecular Biology Grade, \#648466, Calbiochem, Merck, Germany) was used as a detergent. A paraformaldehyde phosphatebuffered solution (PFA, 4\%, 163-20145, Wako Pure Chemical Industries, Ltd., Japan) was used for tissue fixation. An anti-beta actin antibody conjugated with FITC (anti- $\beta$ A FITC Ab, ab6277, mouse monoclonal [AC-15], FITC Ex: 493 nm, Em: 528 nm, Abcam plc., UK) and 4',6-diamidino-2-phenylindole, dihydrochloride (DAPI, Ex: $360 \mathrm{~nm}$, Em: $460 \mathrm{~nm}$, Thermo Fisher Scientific Inc.) were used as fluorescent stains. Sylon CT (5\% dimethyldichlorosilane in toluene, 33065-U, Sigma-Aldrich, Merck) was used as coating on the sensor chip surface. 


\subsection{Apparatus}

The optical arrangement of the parallel-incident-type WGM sensor was reported previously. ${ }^{(7)}$ In this study, a modular USB spectrometer (USB4000, Ocean Optics, FL, USA), a tungsten halogen lamp (HL-2000-FHSA-LL, Ocean Optics), and symmetric-trapezoidal $\mathrm{SiO}_{2}$ prisms were used as a detector, a light source, and a prism of the WGM sensor, respectively.

Figure 1 shows the optical setup of the enhanced fluorescence microscope using WGM illumination. A WGM chip (290-nm-thick $\mathrm{SiO}_{2} / 24$-nm-thick $\mathrm{Si} / 0.725$-mm-thick $\mathrm{SiO}_{2}$ substrate, $14 \times 18 \mathrm{~mm}^{2}$, Shin-Etsu Chemical Co., Ltd., Japan) or a glass chip $\left(0.725\right.$-mm-thick $\mathrm{SiO}_{2}$ substrate, $14 \times 18 \mathrm{~mm}^{2}$ ) was placed on the bottom surface of an asymmetric trapezoidal $\mathrm{SiO}_{2}$ prism with bottom angles of $\alpha$ and $\beta$. At the thicknesses of the $\mathrm{SiO}_{2}$ and $\mathrm{Si}$ layers, the WGM chip can generate enhanced illumination by WGM of which the range of the wavelength is approximately from 600 to $400 \mathrm{~nm}$ when the bottom angle of the prism is from 30 to $40^{\circ}$. The wavelength that can be enhanced by WGM depends on the bottom angle. The surfaces of the chips were illuminated with lights from LEDs ( $\alpha$ side: $490 \mathrm{~nm}$ LED, M490F3, Thorlabs, Inc., NJ, USA; $\beta$ side: 405 nm LED, M405FP1, Thorlabs, Inc.) through optical fibers, collimator lenses, polarizers, short-pass filters ( $\alpha$ side: FESH500, Thorlabs, Inc.; $\beta$ side: FESH450, Thorlabs, Inc.), and the prism. The wavelengths 490 and $405 \mathrm{~nm}$ were selected as the excitation wavelengths of FITC and DAPI in this study, respectively. The incident lights were s-polarized. Long-pass filters (FELH500 and FELH450, Thorlabs, Inc.) were used for eliminating the scattering light. A microscope equipped with an objective lens (Plan Fluor 40×, Nikon Instech Co., Ltd., Japan) and a cooled charge-coupled device camera (BU-59LIR, Bitran Co., Japan) constituted the fluorescence imaging unit. The observation field of the system was limited by the camera used and it was approximately $0.31 \times 0.25 \mathrm{~mm}^{2}$.

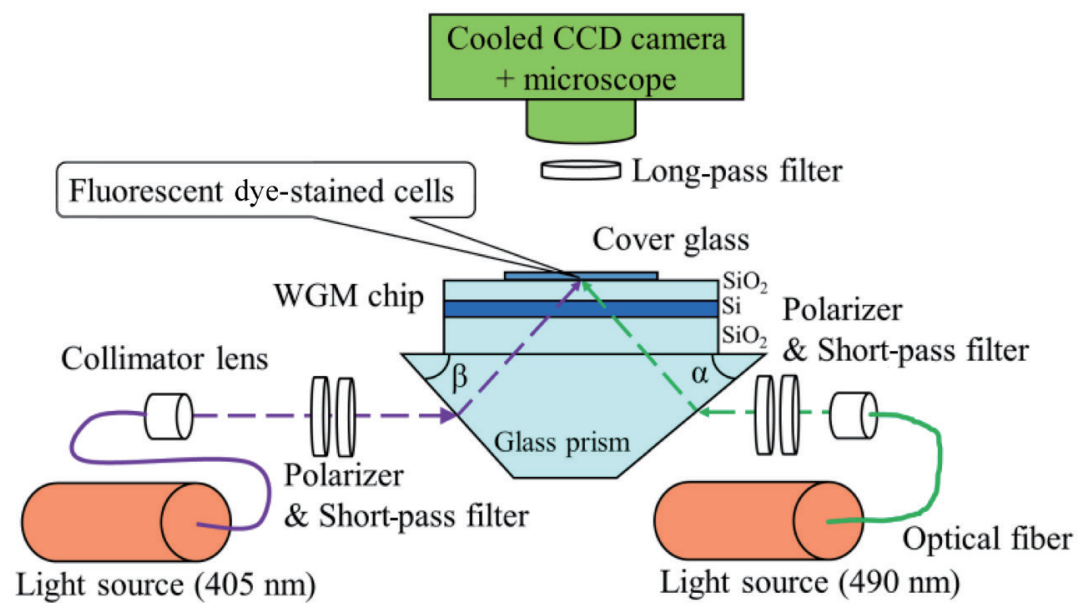

Fig. 1. (Color online) Optical setup of the parallel-incident-type WGM in this study. 


\subsection{Preparation of cultured-cell-containing chips}

The UV-sterilized WGM chip and glass chip were placed in a petri dish. MDCK cells were dissociated with trypsin-EDTA, diluted with DMEM containing 10\% FBS, and poured into the petri dish and cultured. The WGM chip and glass chip were surface-treated with Sylon $\mathrm{CT}$ before use. The incubation period of the cell culture was approximately $36 \mathrm{~h}$. In the case of confluent-cell-containing chips, the incubation period was 5-7 days. After incubation, the WGM chip and glass chip were removed from the petri dish and washed with PBS.

\section{Measurement of Confluent-cell-containing Chip Using WGM Sensor}

The confluent-cultured chip was measured using the WGM sensor. The base angles of the symmetric trapezoidal prisms used in this measurement were 32,35 , and $38^{\circ}$. Simultaneously, the estimated spectra were calculated by the transfer-matrix method for a stratified medium, according to the Fresnel equations. ${ }^{(7,9)}$ In the calculation, the effect of the cell layer on the chip surface was assumed to be equivalent to that of a $60 \mathrm{~nm}$ layer with a refractive index of $1.38^{(10)}$ deposited on the chip surface. Figure 2 shows the reflectance spectra of the confluentcell-containing chip when the base angles were 32,35 , and $38^{\circ}$. Both the measured spectra and estimated spectra of the confluent-cell-containing chip are indicated by solid and dashed lines, respectively. In Fig. 2, the dip positions of the measurement spectra and estimation spectra are almost the same, indicating that the $60 \mathrm{~nm}$ layer with a refractive index of 1.38 is a good approximation of the cultured cells on the chip.

Using the approximation, the bottom angles of the asymmetric trapezoidal prisms $\alpha$ and $\beta$ in Fig. 1 were determined. Estimated reflectance spectra were calculated by the transfer-matrix method when the bottom angle of the prism is from 30 to $45^{\circ}$. According to the spectra, the dip positions were approximately 490 and $405 \mathrm{~nm}$ when the bottom angles were 35 and $40^{\circ}$, respectively. The base angle $\alpha$ was set to $40^{\circ}$ to generate WGM of $405 \mathrm{~nm}$ wavelength, whereas the base angle $\beta$ was set to $35^{\circ}$ to generate WGM of $490 \mathrm{~nm}$ wavelength.

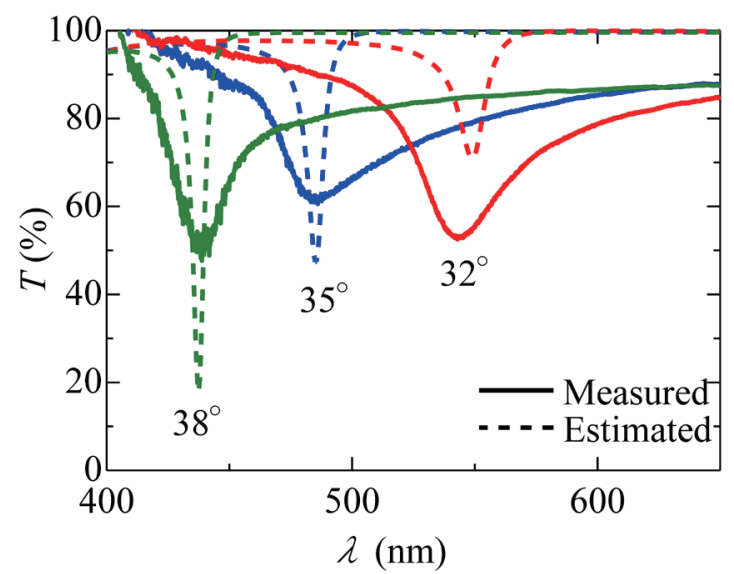

Fig. 2. (Color online) Reflectance spectra with WGM excitation when the base angles of the prisms were 32, 35, and $38^{\circ}$. 


\section{Enhanced Fluorescent Microscopy Using WGM Illumination}

\subsection{Protocol of fluorescence imaging}

To fix the cells and stain the cytoskeleton and nuclei, the cultured-cell-containing chips were immersed in PFA for $15 \mathrm{~min}, 0.1 \%$ Triton X-100 in PBS for $5 \mathrm{~min}, 4.8 \mu \mathrm{g} / \mathrm{mL}$ anti- $\beta A$ FITC Ab for $1 \mathrm{~h}$, and $1 \mu \mathrm{g} / \mathrm{mL}$ DAPI for $10 \mathrm{~min}$. After washing the chips with PBS, cover glasses were placed on the chips and the edges of the cover glasses were sealed with nail polish top coat. A sealed chip was optically attached to the prism via an index matching oil (Code 50350, $n=1.4587$ \pm 0.0005 at $589.3 \mathrm{~nm}$, Cargille-Sacher Laboratories Inc., NJ, USA).

First, light from the $490 \mathrm{~nm}$ LED was irradiated from the $35^{\circ}$ side of the prism, and the cytoskeleton stained with FITC was observed. The exposure time was 3 min. Afterwards, the light source was switched without changing the observation field. Light from the $405 \mathrm{~nm}$ LED was irradiated from the $40^{\circ}$ side of the prism, and the nuclei stained with DAPI were observed. The exposure time was $30 \mathrm{~s}$. These observations were performed using both the WGM chip and the glass chip, and the fluorescence images were compared.

\subsection{Results}

Figure 3 shows photographs of the cytoskeleton stained with FITC observed with the WGM chip (a) and glass chip (b). Monochrome with 16-bit gradation was applied for both images and contrast adjustments were applied individually. The brightness of the background was approximately 1200 in the values of 16-bit luminance. The luminance values of the fields where fluorescence was observed were approximately $2500-10000$ in Fig. 3(a) and 2000-4000 in Fig. 3(b).

Figure 4 shows photographs of the nuclei stained with DAPI observed with the WGM chip (a) and glass chip (b). Monochrome with 16-bit gradation was applied for both images and contrast

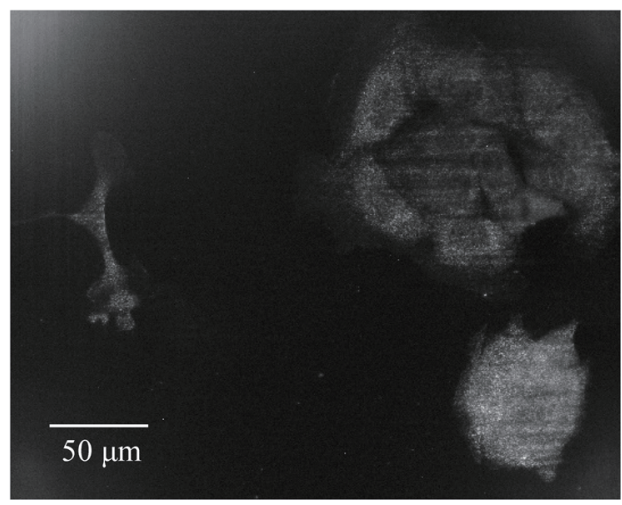

(a)

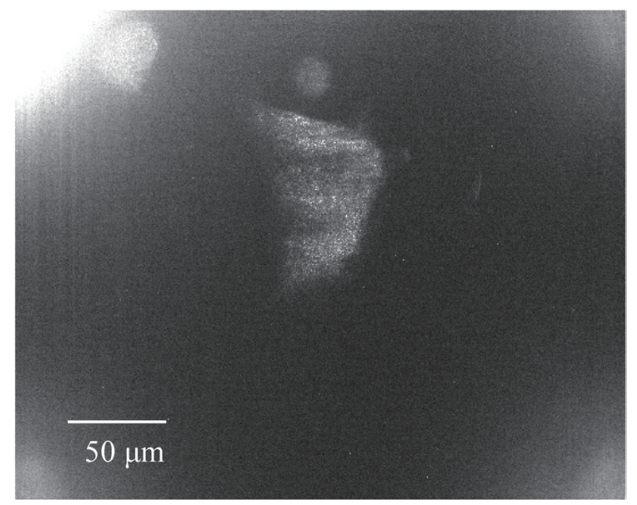

(b)

Fig. 3. Photographs of the cytoskeleton stained with FITC observed with the (a) WGM chip and (b) glass chip. Each observation field was approximately $0.31 \times 0.25 \mathrm{~mm}^{2}$. The exposure time was $3 \mathrm{~min}$. 


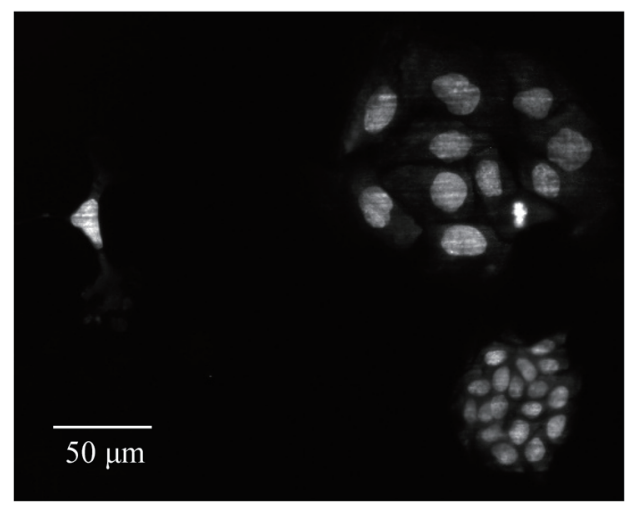

(a)

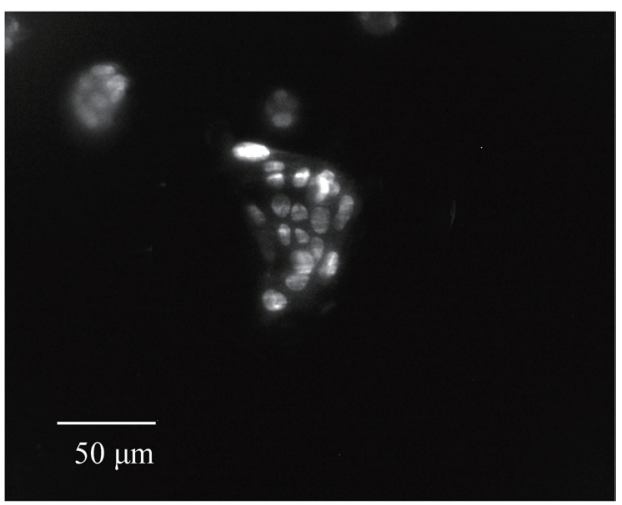

(b)

Fig. 4. Photographs of nuclei stained with DAPI observed with the (a) WGM chip and (b) glass chip. Each observation field was approximately $0.31 \times 0.25 \mathrm{~mm}^{2}$. The exposure time was $30 \mathrm{~s}$.

adjustments were applied individually. The brightness of the background was approximately 1000 in the values of 16-bit luminance. The luminance values of the fields where fluorescence was observed were approximately $20000-58000$ in Fig. 4(a) and 6000-10000 in Fig. 4(b).

\section{Discussion}

Figure 5 shows colored images in which Figs. 3 and 4 are superimposed. The contrast adjustments and colors were applied in the same way in Figs. 5(a) and 5(b). The image editing software GIMP2 (GNU Image Manipulation Program, Ver. 2.8, The GIMP Team) was used for the superposition. Both the luminance value and sharpness of images were higher in the WGM chip, indicating that fluorescence enhancement by WGM worked well in the observation of cells stained with both FITC and DAPI. The maximum values of enhancement were calculated using the luminance values of Figs. 3 and 4. The maximum values of enhancement were defined as ([Maximum value of fluorescence at the WGM chip] - [background])/([Maximum value of fluorescence at the glass chip] - [background]). The maximum value of enhancement with FITC was $(10000-1200) /(4000-1200)$, i.e., approximately 3.1. The maximum value of enhancement with DAPI was $(58000-1000) /(10000$ - 1000), i.e., approximately 6.3 .

In this study, the cultured cells on the chip were regarded to form a $60 \mathrm{~nm}$ layer with a refractive index of 1.38 in the calculation using the transfer-matrix method. By using the approximation, the efficiency of light enhancement on the WGM chip surface covered with the cultured cells was calculated similarly to the simulation using the transfer-matrix method. The efficiencies at wavelengths of 490 and $405 \mathrm{~nm}$ were approximately 70 and 31 times higher, respectively. However, the maximum value of enhancement calculated using luminance values was lower than the estimated values. This can be attributed to the distance of the fluorescentdye-stained cellular tissue from the chip surface, which attenuates the intensity of the electric field of the WGM illumination. 


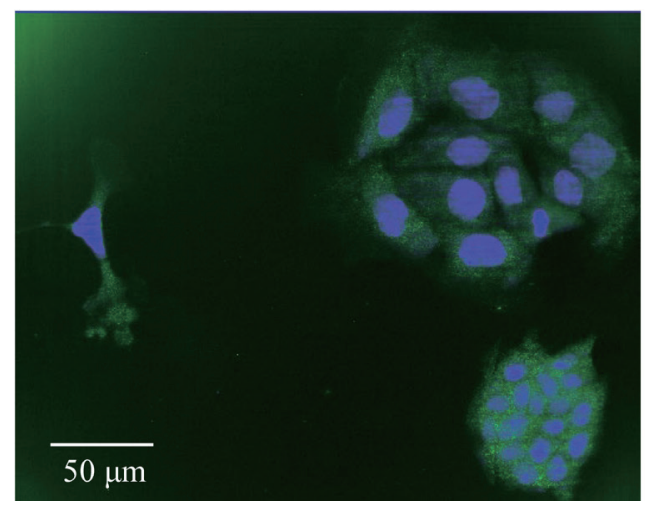

(a)

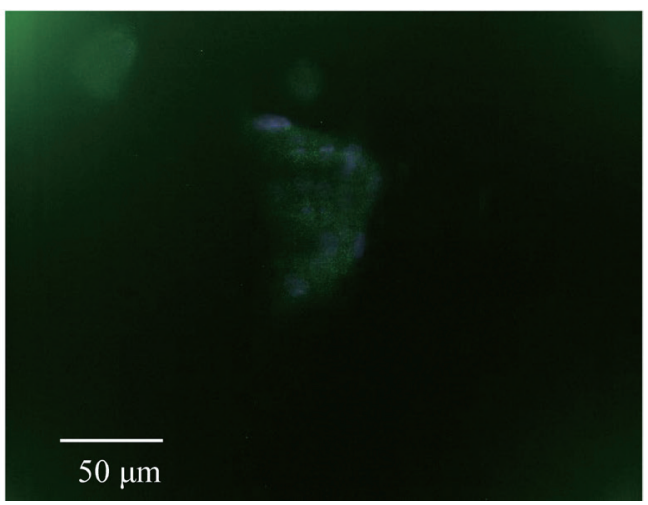

(b)

Fig. 5. (Color online) Colored images in which Figs. 3 and 4 are superimposed observed using (a) the WGM chip and (b) the glass chip. Each observation field was approximately $0.31 \times 0.25 \mathrm{~mm}^{2}$. Figure 3 was colored green, and Fig. 4 was colored purple.

This study is a demonstration of WGM illumination for enhanced fluorescent microscopy. The enhanced fluorescence observations of two colors were performed using the stained cells. This method may be applicable to observations using several fluorescent dyes at the same time by designing a prism that have continuously controllable bottom angles and the optical device. It may also be applicable to fluorescence assays using a microscope.

\section{Conclusions}

In this study, enhanced fluorescence observations using two fluorescent dyes with different excitation wavelengths were performed using WGM illumination. Using the optical arrangement of the parallel-incident-type WGM sensor and asymmetric trapezoidal prism, two wavelengths of WGM illumination were excited on one WGM chip. As a result, enhanced fluorescence observations of two colors were achieved by WGM illumination without moving the observation field of the microscope. Moreover, by designing the shape of the prism and the optical design of the device, we can use several fluorescent dyes at the same time. By this method, we plan to develop WGM field-enhanced multiplex fluorescence immunoassay methods.

\section{Acknowledgments}

This work was partly supported by JSPS KAKENHI grant number 18H01803. We would like to thank the Advanced Functional Materials Research Center of Shin-Etsu Chemical Co., Ltd., for supplying the sensor chip. 


\section{References}

1 H. Schneckenburger: Curr. Opin. Biotechnol. 16 (2005) 13. https://doi.org/10.1016/j.copbio.2004.12.004

2 J. R. Kuhn and T. D. Pollard: Biophys. J. 88 (2005) 1387. https://doi.org/10.1529/biophysj.104.047399

3 T. Liebermann and W. Knoll: Colloids Surf., A 171 (2000) 115. https://doi.org/10.1016/S0927-7757(99)00550-6

4 S. S. Krupka, B. Wiltschi, U. Reuning, K. Hölscher, M. Hara, and E. Sinner: Biosens. Bioelectron. 22 (2006) 260. https://doi.org/10.1016/j.bios.2006.01.004

5 K. Tawa and K. Morigaki: Colloids Surf., B 81 (2010) 447. https://doi.org/10.1016/j.colsurfb.2010.07.038

6 M. Fujimaki, C. Rockstuhl, X. Wang, K. Awazu, J. Tominaga, Y. Koganezawa, Y. Ohki, and T. Komatsubara: Opt. Express 16 (2008) 6408. https://doi.org/10.1364/OE.16.006408

7 M. Fujimaki, X. Wang, T. Kato, K. Awazu, and Y. Ohki: Opt. Express 23 (2015) 10925. https://doi.org/10.1364/ OE.23.010925

8 N. V. Beloglazova, E. S. Speranskaya, A. Wu, Z. Wang, M. Sanders, V. V. Goftman, D. Zhang, I. Yu. Goryacheva, and S. De Saeger: Biosens. Bioelectron. 62 (2014) 59. https://doi.org/10.1016/j.bios.2014.06.021

9 M. Born and E. Wolf: Principles of Optics: Electromagnetic Theory of Propagation, Interference and Diffraction of Light (Pergamon Ltd., Oxford, 1986) 6th ed. reprinted with corrections.

10 B. Kemper, S. Kosmeier, P. Langehanenberg, G. von Bally, I. Bredebusch, W. Domschke, and J. Schnekenburger: J. Biomed. Opt. 12 (2007) 054009-1. https://doi.org/10.1117/1.2798639 